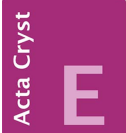
COMMUNICATIONS

ISSN 2056-9890

\section{Crystal structure of 1-(4-fluorophenyl)-4- (4-methoxyphenyl)-1H-1,2,3-triazole}

\author{
Balbir Kumar, ${ }^{\text {a }}$ Madhvi Bhardwaj, ${ }^{\text {b }}$ Satya Paul, ${ }^{\text {b }}$ Rajni \\ Kant $^{\mathrm{a}}$ and Vivek K. Gupta ${ }^{\mathrm{a} *}$
}

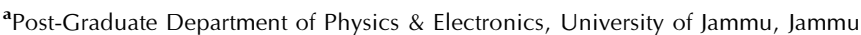
Tawi 180 006, India, and ${ }^{\mathbf{b}}$ Department of Chemistry, University of Jammu, Jammu Tawi 180 006, India. *Correspondence e-mail: vivek_gupta2k2@hotmail.com

Received 11 June 2015; accepted 24 June 2015

Edited by A. J. Lough, University of Toronto, Canada

In the title compound, $\mathrm{C}_{15} \mathrm{H}_{12} \mathrm{FN}_{3} \mathrm{O}$, the triazole ring forms dihedral angles of $30.57(8)$ and $21.81(9)^{\circ}$ with the fluorosubstituted and methoxy-substituted benzene rings, respectively. The dihedral angle between the benzene rings is $51.53(7)^{\circ}$. In the crystal, $\pi-\pi$ interactions between the triazole rings [centroid-centroid seperations $=3.774(2)$ and 3.841 (2) ̊] form chains along [010].

Keywords: crystal structure; 1,2,3-triazole; $\pi-\pi$ interactions.

CCDC reference: 1408544

\section{Related literature}

For related literature on 1,2,3-triazoles, see: Aher et al. (2009); Jordao et al. (2009); Vijaya Raghava Reddy et al. (2010); Soltis et al. (1996). For applications of 1,2,3-triazoles, see: PérezBalderas et al. (2003); Wu et al. (2004); Kumar \& Pandey (2008); Haridas et al. (2008); Turner et al., (2007); Angell \& Burgess (2007); For the synthesis of 1,2,3-triazoles, see: Huisgen et al. (1965); Wang et al. (2010). For related structures, see: Abdel-Wahab et al. (2012); Zhang et al. (2004).

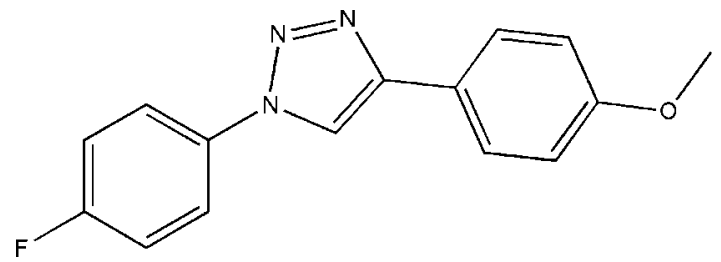

\section{Experimental}

2.1. Crystal data

$\mathrm{C}_{15} \mathrm{H}_{12} \mathrm{FN}_{3} \mathrm{O} \quad M_{r}=269.28$
Triclinic, $P \overline{1}$

$a=5.6572(5) \AA$

$b=7.3692(8) \AA$

$c=15.5711(15) \AA$

$\alpha=79.202$ (9)

$\beta=81.159$ (8)

$\gamma=89.442(8)^{\circ}$

\subsection{Data collection}

Oxford Diffraction Xcalibur Sapphire3 diffractometer

Absorption correction: multi-scan

(CrysAlis PRO; Oxford

Diffraction, 2010)

$T_{\text {min }}=0.806, T_{\max }=1.000$

\subsection{Refinement}

$R\left[F^{2}>2 \sigma\left(F^{2}\right)\right]=0.057$

$w R\left(F^{2}\right)=0.179$

$S=1.04$

2461 reflections

$$
\begin{aligned}
& V=629.95(11) \AA^{3} \\
& Z=2 \\
& \text { Mo } K \alpha \text { radiation } \\
& \mu=0.10 \mathrm{~mm}^{-1} \\
& T=293 \mathrm{~K} \\
& 0.30 \times 0.20 \times 0.20 \mathrm{~mm}
\end{aligned}
$$

4369 measured reflections 2461 independent reflections 1575 reflections with $I>2 \sigma(I)$ $R_{\text {int }}=0.034$

Data collection: CrysAlis PRO (Oxford Diffraction, 2010); cell refinement: CrysAlis PRO; data reduction: CrysAlis PRO; program(s) used to solve structure: SHELXS97 (Sheldrick, 2008); program(s) used to refine structure: SHELXL97 (Sheldrick, 2008); molecular graphics: ORTEP-3 for Windows (Farrugia, 2012); software used to prepare material for publication: PLATON (Spek, 2009).

\title{
Acknowledgements
}

RK acknowledges the Department of Science \& Technology for the single-crystal X-ray diffractometer sanctioned as a National Facility under Project No. SR/S2/CMP-47/2003.

Supporting information for this paper is available from the IUCr electronic archives (Reference: LH5772).

\section{References}

Abdel-Wahab, B. F., Mohamed, H. A., Ng, S. W. \& Tiekink, E. R. T. (2012) Acta Cryst. E68, o1956-01957.

Aher, N. G., Pore, V. S., Mishra, N. N., Kumar, A., Shukla, P. K., Sharma, A. \& Bhat, M. K. (2009). Bioorg. Med. Chem. Lett. 19, 759-763.

Angell, Y. \& Burgess, K. (2007). Chem. Soc. Rev. 36, 1674-1689.

Farrugia, L. J. (2012). J. Appl. Cryst. 45, 849-854.

Haridas, V., Lal, K., Sharma, Y. K. \& Upreti, S. (2008). Org. Lett. 10, 16451647.

Huisgen, R., Knorr, R., Möbius, L. \& Szeimies, G. (1965). Chem. Ber. 98, 4014 4021.

Jordão, A. K., Ferreira, V. F., Lima, E. S., de Souza, M. C. B. V., Carlos, E. C. L., Castro, H. C., Geraldo, R. B., Rodrigues, C. R., Almeida, M. C. B. \& Cunha, A. C. (2009). Bioorg. Med. Chem. 17, 3713-3719.

Kumar, A. \& Pandey, P. S. (2008). Org. Lett. 10, 165-168.

Oxford Diffraction (2010). CrysAlis PRO. Oxford Diffraction Ltd, Yarnton, England.

Pérez-Balderas, F., Ortega-Muñoz, M., Morales-Sanfrutos, J., HernándezMateo, F., Calvo-Flores, F. G., Calvo-Asín, J. A., Isac-García, J. \& SantoyoGonzález, F. (2003). Org. Lett. 5, 1951-1954.

Sheldrick, G. M. (2008). Acta Cryst. A64, 112-122.

Soltis, M. J., Yeh, H. J., Cole, K. A., Whittaker, N., Wersto, R. P. \& Kohn, E. C (1996). Drug Metab. Dispos. 24, 799-806.

Spek, A. L. (2009). Acta Cryst. D65, 148-155. 


\section{data reports}

Turner, R. A., Oliver, A. G. \& Lokey, R. S. (2007). Org. Lett. 9, 5011-5014.

Vijaya Raghava Reddy, L., Venkat Reddy, P., Mishra, N. N., Shukla, P. K., Yadav, G., Srivastava, R. \& Shaw, A. K. (2010). Carbohydr. Res. 345, 15151521.

Wang, D., Li, N., Zhao, M., Shi, W., Ma, C. \& Chen, B. (2010). Green Chem. 12, 2120 .
Wu, P., Feldman, A. K., Nugent, A. K., Hawker, C. J., Scheel, A., Voit, B., Pyun, J., Fréchet, J. M. J., Sharpless, K. B. \& Fokin, V. V. (2004). Angew. Chem. Int. Ed. 43, 3928-3932.

Zhang, L.-X., Zhang, A.-J., Lei, X.-X., Zou, K.-H. \& Ng, S. W. (2004). Acta Cryst. E60, o613-0615. 


\title{
supporting information
}

Acta Cryst. (2015). E71, 0534-o535 [https://doi.org/10.1107/S2056989015012153]

\section{Crystal structure of 1-(4-fluorophenyl)-4-(4-methoxyphenyl)-1 H-1,2,3-triazole}

\author{
Balbir Kumar, Madhvi Bhardwaj, Satya Paul, Rajni Kant and Vivek K. Gupta
}

\section{S1. Comment}

1,2,3-Triazoles are an important class of organic compounds which have become prominent in recent years as superbly versatile five membered nitrogen heterocycles. The 1,2,3-triazole family exhibit a broad spectrum of bioactivities such as antifungal (Aher et al., 2009) antiviral (Jordao et al., 2009), antibacterial (Vijaya Raghava Reddy et al., 2010) and anticancer (Soltis et al., 1996) activities. Furthermore 1,4-disubstituted 1,2,3-triazoles have also been used as a ligation tool for the synthesis of neoglyco-conjugates (Perez-Balderas et al., 2003), multivalent dendrimeric peptides (Wu et al., 2004), ionic receptors (Kumar et al., 2008), triazolophanes (Haridas et al., 2008), cyclic peptides (Turner et al., 2007) and peptidomimetics (Angell et al., 2007). 1,2,3-Triazoles are traditionally obtained using the thermal 1,3-dipolar cycloaddition of organic azides with alkynes (Huisgen et al., 1965) that has been known for nearly five decades. Recently, copper based catalysis was found to dramatically accelerate the reaction under mild conditions while achieving a high regioselectivity towards the 1,4-regioisomer of the triazole product (Wang et al., 2010). This powerful, highly reliable, and selective reaction is the paradigm of a click reaction, which placed it in a class of its own and has enabled many novel applications.

The molecular structure of the title compound is shown in Fig. 1. The triazole ring forms dihedral angles of $30.57(8)^{\circ}$ and $21.81(9)^{\circ}$ with the fluoro-substituted and methoxy-substituted benzen rings, respectively. The dihedral angle between the benzene rings is $51.53(7)^{\circ}$. All bond lengths and angles are normal and correspond to those observed in the related structures (Zhang et al., 2004; Abdel-Wahab et al., 2012). The C15-F1 bond length [1.357 (4) §] agrees well with the accepted value of $1.340 \AA$ for the $\mathrm{F}-\mathrm{C}_{\text {aromatic }}$ length and is in good agreement with a structure of this type (AbdelWahab et al., 2012). In the crystal, $\pi-\pi$ interactions observed between the triazole rings [centroid-centroid seperations $=$ 3.774 (2) and 3.841 (2) §] form chains along [010] (Fig. 2).

\section{S2. Experimental}

Synthesis of 1-(4-flourophenyl)-4-(4-methoxyphenyl) -1H-1,2,3-triazole: To 4-fluoroaniline ( $0.22 \mathrm{~g}, 2 \mathrm{mmol})$ in a round bottomed flask maintained at 273-278 K, mixture of conc. $\mathrm{HCl}: \mathrm{H}_{2} \mathrm{O}(1.5 \mathrm{ml}, 1: 1)$ was added and stirred for $5 \mathrm{~min}$. Then solution of $\mathrm{NaNO}_{2}(0.17 \mathrm{~g}, 2.5 \mathrm{mmol}$ in $1 \mathrm{ml}$ water $)$ was added dropwise over a period of $5 \mathrm{~min}$. After stirring for another $5 \mathrm{~min}$, sodium azide $(0.19 \mathrm{~g}, 3 \mathrm{mmol})$ was added and the reaction mixture was further stirred for another $10 \mathrm{~min}$. Finally, 4-methoxyphenylacetylene $(0.19 \mathrm{~g}, 1.5 \mathrm{mmol})$ and catalyst $\left[\mathrm{Cu}(0)-\mathrm{Fe}_{3} \mathrm{O}_{4} @ \mathrm{SiO}_{2} / \mathrm{NH}_{2} \mathrm{Cel}\right](0.05 \mathrm{~g})$ were added to the reaction mixture followed by stirring at room temperature for $6 \mathrm{~h}$. The reaction was then stopped and the catalyst was separated using an external magnet. The reaction mixture was extracted with EtOAc, washed with water and dried over $\mathrm{Na}_{2} \mathrm{SO}_{4}$. Finally, the product was obtained after removal of the solvent under reduced pressure followed by crystallization with EtOAc: pet ether. The product, 1-(4-flourophenyl)-4-(4-methoxyphenyl) -1H-1,2,3-triazole was obtained as shiny white crystals. 


\section{S3. Refinement}

All $\mathrm{H}$ atoms were geometrically fixed and allowed to ride on their parent $\mathrm{C}$ atoms, with $\mathrm{C}-\mathrm{H}$ distances of $0.93-0.96 \AA$; and with $U_{\text {iso }}(\mathrm{H})=1.2 U_{\text {eq }}(\mathrm{C})$, except for the methyl group where $U_{\text {iso }}(\mathrm{H})=1.5 U_{\text {eq }}(\mathrm{C})$.

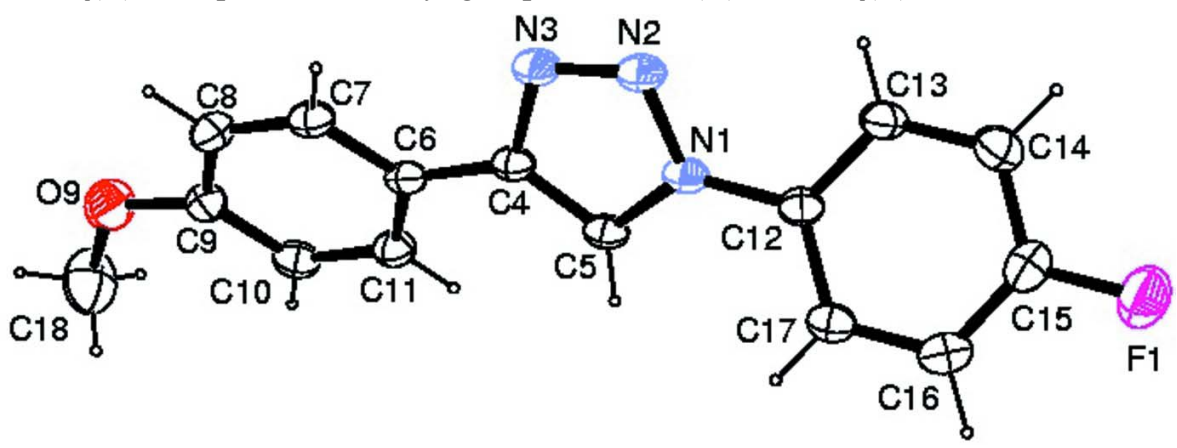

Figure 1

The molecular structure of the title compound with displacement ellipsoids drawn at the $40 \%$ probability level. $\mathrm{H}$ atoms are shown as small spheres of arbitrary radii. 


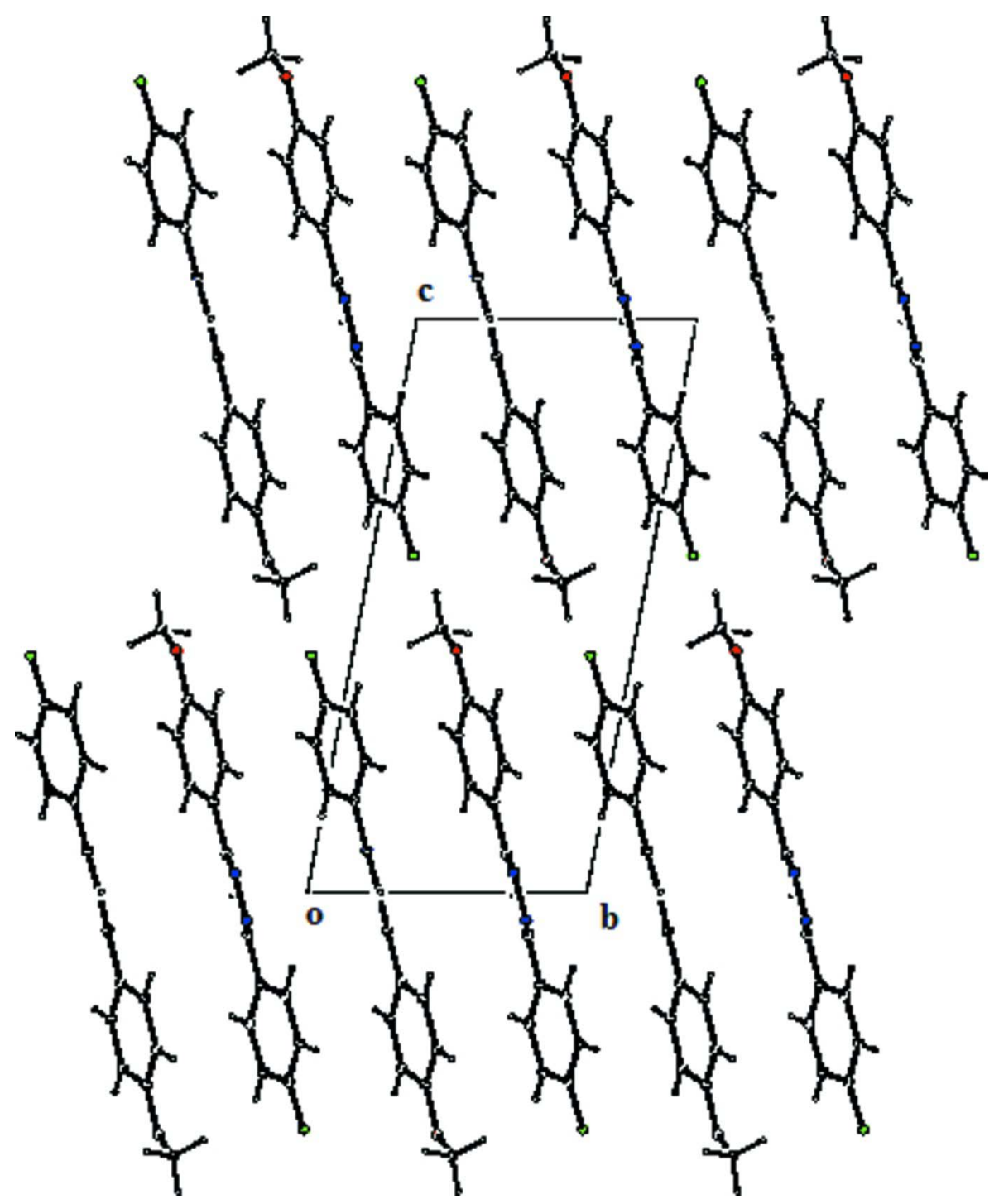

Figure 2

The packing arrangement of molecules viewed along the $a$ axis.

1-(4-Fluorophenyl)-4-(4-methoxyphenyl)-1H-1,2,3-triazole

Crystal data

$\mathrm{C}_{15} \mathrm{H}_{12} \mathrm{FN}_{3} \mathrm{O}$

$M_{r}=269.28$

Triclinic, $P \overline{1}$

Hall symbol: -P 1

$a=5.6572(5) \AA$

$b=7.3692(8) \AA$

$c=15.5711(15) \AA$

$\alpha=79.202(9)^{\circ}$

$\beta=81.159(8)^{\circ}$

$\gamma=89.442(8)^{\circ}$

$V=629.95(11) \AA^{3}$

$Z=2$

$$
\begin{aligned}
& F(000)=280 \\
& D_{\mathrm{x}}=1.420 \mathrm{Mg} \mathrm{m}^{-3} \\
& D_{\mathrm{m}}=1.42 \mathrm{Mg} \mathrm{m}^{-3} \\
& D_{\mathrm{m}} \text { measured by not measured } \\
& \text { Mo } K \alpha \text { radiation, } \lambda=0.71073 \AA \\
& \text { Cell parameters from } 1205 \text { reflections } \\
& \theta=4.0-28.0^{\circ} \\
& \mu=0.10 \mathrm{~mm}^{-1} \\
& T=293 \mathrm{~K} \\
& \text { Block, white } \\
& 0.30 \times 0.20 \times 0.20 \mathrm{~mm}
\end{aligned}
$$




\section{Data collection}

Oxford Diffraction Xcalibur Sapphire3 diffractometer

Radiation source: fine-focus sealed tube Graphite monochromator

Detector resolution: 16.1049 pixels $\mathrm{mm}^{-1}$ $\omega$ scans

Absorption correction: multi-scan

(CrysAlis PRO; Oxford Diffraction, 2010)

$T_{\min }=0.806, T_{\max }=1.000$

Refinement

Refinement on $F^{2}$

Least-squares matrix: full

$R\left[F^{2}>2 \sigma\left(F^{2}\right)\right]=0.057$

$w R\left(F^{2}\right)=0.179$

$S=1.04$

2461 reflections

182 parameters

0 restraints

Primary atom site location: structure-invariant direct methods
4369 measured reflections

2461 independent reflections

1575 reflections with $I>2 \sigma(I)$

$R_{\text {int }}=0.034$

$\theta_{\text {max }}=26.0^{\circ}, \theta_{\min }=3.7^{\circ}$

$h=-4 \rightarrow 6$

$k=-7 \rightarrow 9$

$l=-18 \rightarrow 19$

Secondary atom site location: difference Fourier map

Hydrogen site location: inferred from neighbouring sites

$\mathrm{H}$-atom parameters constrained

$w=1 /\left[\sigma^{2}\left(F_{\mathrm{o}}{ }^{2}\right)+(0.0744 P)^{2}+0.0652 P\right]$

where $P=\left(F_{\mathrm{o}}^{2}+2 F_{\mathrm{c}}^{2}\right) / 3$

$(\Delta / \sigma)_{\max }<0.001$

$\Delta \rho_{\max }=0.26 \mathrm{e} \AA^{-3}$

$\Delta \rho_{\text {min }}=-0.22$ e $\AA^{-3}$

\section{Special details}

Geometry. All e.s.d.'s (except the e.s.d. in the dihedral angle between two 1.s. planes) are estimated using the full covariance matrix. The cell e.s.d.'s are taken into account individually in the estimation of e.s.d.'s in distances, angles and torsion angles; correlations between e.s.d.'s in cell parameters are only used when they are defined by crystal symmetry. An approximate (isotropic) treatment of cell e.s.d.'s is used for estimating e.s.d.'s involving 1.s. planes.

Refinement. Refinement of $F^{2}$ against ALL reflections. The weighted $R$-factor $w R$ and goodness of fit $S$ are based on $F^{2}$, conventional $R$-factors $R$ are based on $F$, with $F$ set to zero for negative $F^{2}$. The threshold expression of $F^{2}>\sigma\left(F^{2}\right)$ is used only for calculating $R$-factors (gt) etc. and is not relevant to the choice of reflections for refinement. $R$-factors based on $F^{2}$ are statistically about twice as large as those based on $F$, and $R$ - factors based on ALL data will be even larger.

Fractional atomic coordinates and isotropic or equivalent isotropic displacement parameters $\left(\AA^{2}\right)$

\begin{tabular}{lllll}
\hline & $x$ & $y$ & $z$ & $U_{\text {iss }} * / U_{\text {eq }}$ \\
\hline F1 & $0.2052(4)$ & $-0.1487(2)$ & $0.41281(12)$ & $0.0782(6)$ \\
N1 & $0.1232(4)$ & $0.1852(3)$ & $0.07275(13)$ & $0.0404(5)$ \\
N2 & $-0.0970(4)$ & $0.1982(3)$ & $0.04842(15)$ & $0.0505(6)$ \\
N3 & $-0.0714(4)$ & $0.2743(3)$ & $-0.03467(15)$ & $0.0489(6)$ \\
C4 & $0.1657(4)$ & $0.3132(3)$ & $-0.06593(16)$ & $0.0367(6)$ \\
C5 & $0.2893(4)$ & $0.2552(3)$ & $0.00235(15)$ & $0.0395(6)$ \\
H5 & 0.4540 & 0.2623 & 0.0009 & $0.047^{*}$ \\
C6 & $0.2436(4)$ & $0.3971(3)$ & $-0.15737(16)$ & $0.0371(6)$ \\
C7 & $0.1015(5)$ & $0.3849(3)$ & $-0.22101(17)$ & $0.0426(6)$ \\
H7 & -0.0454 & 0.3228 & -0.2040 & $0.051^{*}$ \\
C8 & $0.1707(5)$ & $0.4613(4)$ & $-0.30769(18)$ & $0.0483(7)$ \\
H8 & 0.0717 & 0.4501 & -0.3490 & $0.058^{*}$ \\
C9 & $0.3880(5)$ & $0.5558(3)$ & $-0.33479(17)$ & $0.0457(7)$ \\
O9 & $0.4360(4)$ & $0.6328(3)$ & $-0.42195(14)$ & $0.0718(7)$ \\
C10 & $0.5341(5)$ & $0.5698(3)$ & $-0.27319(17)$ & $0.0452(6)$ \\
H10 & 0.6804 & 0.6327 & -0.2906 & $0.054^{*}$
\end{tabular}




$\begin{array}{lllll}\text { C11 } & 0.4626(5) & 0.4900(3) & -0.18530(16) & 0.0414(6) \\ \text { H11 } & 0.5629 & 0.4987 & -0.1441 & 0.050^{*} \\ \text { C12 } & 0.1484(4) & 0.1035(3) & 0.16056(16) & 0.0371(6) \\ \text { C13 } & -0.0340(5) & 0.1177(3) & 0.22842(16) & 0.0430(6) \\ \text { H13 } & -0.1694 & 0.1844 & 0.2166 & 0.052^{*} \\ \text { C14 } & -0.0162(5) & 0.0338(4) & 0.31322(18) & 0.0518(7) \\ \text { H14 } & -0.1391 & 0.0417 & 0.3594 & 0.062^{*} \\ \text { C15 } & 0.1878(5) & -0.0630(3) & 0.32897(18) & 0.0500(7) \\ \text { C16 } & 0.3719(5) & -0.0751(3) & 0.26282(19) & 0.0503(7) \\ \text { H16 } & 0.5088 & -0.1392 & 0.2752 & 0.060^{*} \\ \text { C17 } & 0.3531(5) & 0.0081(3) & 0.17797(17) & 0.0425(6) \\ \text { H17 } & 0.4773 & 0.0007 & 0.1322 & 0.051^{*} \\ \text { C18 } & 0.6617(8) & 0.6997(5) & -0.4596(2) & 0.0918(12) \\ \text { H18A } & 0.7031 & 0.7988 & -0.4321 & 0.138^{*} \\ \text { H18B } & 0.6648 & 0.7446 & -0.5218 & 0.138^{*} \\ \text { H18C } & 0.7749 & 0.6025 & -0.4512 & 0.138^{*}\end{array}$

Atomic displacement parameters $\left(\AA^{2}\right)$

\begin{tabular}{lllllll}
\hline & $U^{11}$ & $U^{22}$ & $U^{33}$ & $U^{12}$ & $U^{13}$ & $U^{23}$ \\
\hline F1 & $0.0830(15)$ & $0.0859(12)$ & $0.0607(12)$ & $0.0042(11)$ & $-0.0198(10)$ & $0.0062(9)$ \\
N1 & $0.0268(11)$ & $0.0459(12)$ & $0.0481(13)$ & $0.0005(9)$ & $-0.0039(9)$ & $-0.0096(9)$ \\
N2 & $0.0252(11)$ & $0.0691(14)$ & $0.0558(15)$ & $-0.0006(10)$ & $-0.0048(10)$ & $-0.0098(11)$ \\
N3 & $0.0260(12)$ & $0.0675(15)$ & $0.0508(14)$ & $-0.0006(11)$ & $-0.0033(10)$ & $-0.0069(11)$ \\
C4 & $0.0268(13)$ & $0.0389(12)$ & $0.0455(15)$ & $0.0003(10)$ & $-0.0056(10)$ & $-0.0105(10)$ \\
C5 & $0.0224(12)$ & $0.0470(14)$ & $0.0483(15)$ & $-0.0018(11)$ & $-0.0025(10)$ & $-0.0090(11)$ \\
C6 & $0.0279(13)$ & $0.0391(12)$ & $0.0458(15)$ & $0.0054(10)$ & $-0.0075(11)$ & $-0.0108(10)$ \\
C7 & $0.0303(13)$ & $0.0450(13)$ & $0.0536(16)$ & $-0.0001(11)$ & $-0.0086(11)$ & $-0.0107(11)$ \\
C8 & $0.0393(15)$ & $0.0583(16)$ & $0.0513(17)$ & $0.0049(13)$ & $-0.0158(13)$ & $-0.0136(12)$ \\
C9 & $0.0450(16)$ & $0.0499(14)$ & $0.0404(15)$ & $0.0101(13)$ & $-0.0063(12)$ & $-0.0049(11)$ \\
O9 & $0.0608(14)$ & $0.0911(15)$ & $0.0542(13)$ & $-0.0043(12)$ & $-0.0021(11)$ & $0.0045(11)$ \\
C10 & $0.0321(14)$ & $0.0458(14)$ & $0.0553(17)$ & $-0.0029(12)$ & $0.0001(12)$ & $-0.0083(12)$ \\
C11 & $0.0331(14)$ & $0.0447(13)$ & $0.0484(15)$ & $0.0003(11)$ & $-0.0088(11)$ & $-0.0120(11)$ \\
C12 & $0.0293(13)$ & $0.0358(12)$ & $0.0458(15)$ & $-0.0034(10)$ & $-0.0048(11)$ & $-0.0070(10)$ \\
C13 & $0.0316(13)$ & $0.0452(14)$ & $0.0497(16)$ & $0.0032(11)$ & $-0.0020(11)$ & $-0.0063(11)$ \\
C14 & $0.0441(16)$ & $0.0556(16)$ & $0.0522(18)$ & $0.0000(14)$ & $0.0027(13)$ & $-0.0092(12)$ \\
C15 & $0.0532(18)$ & $0.0471(15)$ & $0.0493(17)$ & $-0.0049(13)$ & $-0.0165(14)$ & $-0.0011(12)$ \\
C16 & $0.0401(15)$ & $0.0457(14)$ & $0.0657(19)$ & $0.0059(12)$ & $-0.0131(14)$ & $-0.0079(13)$ \\
C17 & $0.0317(13)$ & $0.0425(13)$ & $0.0535(16)$ & $0.0001(11)$ & $-0.0038(11)$ & $-0.0121(11)$ \\
C18 & $0.091(3)$ & $0.109(3)$ & $0.068(2)$ & $-0.032(2)$ & $0.000(2)$ & $-0.0060(19)$ \\
& & & & & & \\
\hline
\end{tabular}

Geometric parameters $\left(A,{ }^{\circ}\right)$

\begin{tabular}{llll}
\hline $\mathrm{F} 1-\mathrm{C} 15$ & $1.357(3)$ & $\mathrm{O} 9-\mathrm{C} 18$ & $1.376(4)$ \\
$\mathrm{N} 1-\mathrm{C} 5$ & $1.354(3)$ & $\mathrm{C} 10-\mathrm{C} 11$ & $1.384(3)$ \\
$\mathrm{N} 1-\mathrm{N} 2$ & $1.354(3)$ & $\mathrm{C} 10-\mathrm{H} 10$ & 0.9300 \\
$\mathrm{~N} 1-\mathrm{C} 12$ & $1.415(3)$ & $\mathrm{C} 11-\mathrm{H} 11$ & 0.9300 \\
$\mathrm{~N} 2-\mathrm{N} 3$ & $1.297(3)$ & $\mathrm{C} 12-\mathrm{C} 13$ & $1.377(3)$
\end{tabular}




\begin{tabular}{|c|c|c|c|}
\hline $\mathrm{N} 3-\mathrm{C} 4$ & $1.368(3)$ & $\mathrm{C} 12-\mathrm{C} 17$ & $1.385(3)$ \\
\hline $\mathrm{C} 4-\mathrm{C} 5$ & $1.362(3)$ & $\mathrm{C} 13-\mathrm{C} 14$ & $1.367(3)$ \\
\hline $\mathrm{C} 4-\mathrm{C} 6$ & $1.444(3)$ & $\mathrm{C} 13-\mathrm{H} 13$ & 0.9300 \\
\hline $\mathrm{C} 5-\mathrm{H} 5$ & 0.9300 & $\mathrm{C} 14-\mathrm{C} 15$ & $1.381(4)$ \\
\hline $\mathrm{C} 6-\mathrm{C} 7$ & $1.384(3)$ & $\mathrm{C} 14-\mathrm{H} 14$ & 0.9300 \\
\hline $\mathrm{C} 6-\mathrm{C} 11$ & $1.390(3)$ & $\mathrm{C} 15-\mathrm{C} 16$ & $1.363(4)$ \\
\hline $\mathrm{C} 7-\mathrm{C} 8$ & $1.360(3)$ & $\mathrm{C} 16-\mathrm{C} 17$ & $1.368(3)$ \\
\hline $\mathrm{C} 7-\mathrm{H} 7$ & 0.9300 & $\mathrm{C} 16-\mathrm{H} 16$ & 0.9300 \\
\hline $\mathrm{C} 8-\mathrm{C} 9$ & $1.387(4)$ & C17-H17 & 0.9300 \\
\hline $\mathrm{C} 8-\mathrm{H} 8$ & 0.9300 & C18-H18A & 0.9600 \\
\hline $\mathrm{C} 9-\mathrm{O} 9$ & $1.356(3)$ & C18-H18B & 0.9600 \\
\hline $\mathrm{C} 9-\mathrm{C} 10$ & $1.377(4)$ & $\mathrm{C} 18-\mathrm{H} 18 \mathrm{C}$ & 0.9600 \\
\hline $\mathrm{C} 5-\mathrm{N} 1-\mathrm{N} 2$ & $109.5(2)$ & $\mathrm{C} 10-\mathrm{C} 11-\mathrm{C} 6$ & $121.3(2)$ \\
\hline $\mathrm{C} 5-\mathrm{N} 1-\mathrm{C} 12$ & $130.8(2)$ & $\mathrm{C} 10-\mathrm{C} 11-\mathrm{H} 11$ & 119.4 \\
\hline $\mathrm{N} 2-\mathrm{N} 1-\mathrm{C} 12$ & $119.7(2)$ & $\mathrm{C} 6-\mathrm{C} 11-\mathrm{H} 11$ & 119.4 \\
\hline $\mathrm{N} 3-\mathrm{N} 2-\mathrm{N} 1$ & $107.7(2)$ & $\mathrm{C} 13-\mathrm{C} 12-\mathrm{C} 17$ & $120.3(2)$ \\
\hline $\mathrm{N} 2-\mathrm{N} 3-\mathrm{C} 4$ & $109.6(2)$ & $\mathrm{C} 13-\mathrm{C} 12-\mathrm{N} 1$ & $119.3(2)$ \\
\hline $\mathrm{C} 5-\mathrm{C} 4-\mathrm{N} 3$ & $107.4(2)$ & $\mathrm{C} 17-\mathrm{C} 12-\mathrm{N} 1$ & $120.4(2)$ \\
\hline $\mathrm{C} 5-\mathrm{C} 4-\mathrm{C} 6$ & $131.8(2)$ & $\mathrm{C} 14-\mathrm{C} 13-\mathrm{C} 12$ & $120.1(2)$ \\
\hline $\mathrm{N} 3-\mathrm{C} 4-\mathrm{C} 6$ & $120.8(2)$ & $\mathrm{C} 14-\mathrm{C} 13-\mathrm{H} 13$ & 120.0 \\
\hline $\mathrm{N} 1-\mathrm{C} 5-\mathrm{C} 4$ & $105.8(2)$ & $\mathrm{C} 12-\mathrm{C} 13-\mathrm{H} 13$ & 120.0 \\
\hline $\mathrm{N} 1-\mathrm{C} 5-\mathrm{H} 5$ & 127.1 & $\mathrm{C} 13-\mathrm{C} 14-\mathrm{C} 15$ & $118.7(2)$ \\
\hline $\mathrm{C} 4-\mathrm{C} 5-\mathrm{H} 5$ & 127.1 & $\mathrm{C} 13-\mathrm{C} 14-\mathrm{H} 14$ & 120.7 \\
\hline $\mathrm{C} 7-\mathrm{C} 6-\mathrm{C} 11$ & $117.5(2)$ & $\mathrm{C} 15-\mathrm{C} 14-\mathrm{H} 14$ & 120.7 \\
\hline $\mathrm{C} 7-\mathrm{C} 6-\mathrm{C} 4$ & $120.5(2)$ & $\mathrm{F} 1-\mathrm{C} 15-\mathrm{C} 16$ & $119.1(2)$ \\
\hline $\mathrm{C} 11-\mathrm{C} 6-\mathrm{C} 4$ & $122.0(2)$ & $\mathrm{F} 1-\mathrm{C} 15-\mathrm{C} 14$ & $119.0(3)$ \\
\hline $\mathrm{C} 8-\mathrm{C} 7-\mathrm{C} 6$ & $121.9(3)$ & $\mathrm{C} 16-\mathrm{C} 15-\mathrm{C} 14$ & $121.9(3)$ \\
\hline $\mathrm{C} 8-\mathrm{C} 7-\mathrm{H} 7$ & 119.1 & $\mathrm{C} 15-\mathrm{C} 16-\mathrm{C} 17$ & $119.2(2)$ \\
\hline $\mathrm{C} 6-\mathrm{C} 7-\mathrm{H} 7$ & 119.1 & $\mathrm{C} 15-\mathrm{C} 16-\mathrm{H} 16$ & 120.4 \\
\hline $\mathrm{C} 7-\mathrm{C} 8-\mathrm{C} 9$ & $120.3(2)$ & $\mathrm{C} 17-\mathrm{C} 16-\mathrm{H} 16$ & 120.4 \\
\hline $\mathrm{C} 7-\mathrm{C} 8-\mathrm{H} 8$ & 119.9 & $\mathrm{C} 16-\mathrm{C} 17-\mathrm{C} 12$ & $119.7(2)$ \\
\hline $\mathrm{C} 9-\mathrm{C} 8-\mathrm{H} 8$ & 119.9 & $\mathrm{C} 16-\mathrm{C} 17-\mathrm{H} 17$ & 120.1 \\
\hline $\mathrm{O} 9-\mathrm{C} 9-\mathrm{C} 10$ & $125.0(3)$ & $\mathrm{C} 12-\mathrm{C} 17-\mathrm{H} 17$ & 120.1 \\
\hline $\mathrm{O} 9-\mathrm{C} 9-\mathrm{C} 8$ & $115.6(3)$ & $\mathrm{O} 9-\mathrm{C} 18-\mathrm{H} 18 \mathrm{~A}$ & 109.5 \\
\hline $\mathrm{C} 10-\mathrm{C} 9-\mathrm{C} 8$ & $119.3(2)$ & $\mathrm{O} 9-\mathrm{C} 18-\mathrm{H} 18 \mathrm{~B}$ & 109.5 \\
\hline $\mathrm{C} 9-\mathrm{O} 9-\mathrm{C} 18$ & $120.7(3)$ & $\mathrm{H} 18 \mathrm{~A}-\mathrm{C} 18-\mathrm{H} 18 \mathrm{~B}$ & 109.5 \\
\hline $\mathrm{C} 9-\mathrm{C} 10-\mathrm{C} 11$ & $119.8(3)$ & $\mathrm{O} 9-\mathrm{C} 18-\mathrm{H} 18 \mathrm{C}$ & 109.5 \\
\hline $\mathrm{C} 9-\mathrm{C} 10-\mathrm{H} 10$ & 120.1 & $\mathrm{H} 18 \mathrm{~A}-\mathrm{C} 18-\mathrm{H} 18 \mathrm{C}$ & 109.5 \\
\hline $\mathrm{C} 11-\mathrm{C} 10-\mathrm{H} 10$ & 120.1 & $\mathrm{H} 18 \mathrm{~B}-\mathrm{C} 18-\mathrm{H} 18 \mathrm{C}$ & 109.5 \\
\hline $\mathrm{C} 5-\mathrm{N} 1-\mathrm{N} 2-\mathrm{N} 3$ & $-0.1(3)$ & $\mathrm{O} 9-\mathrm{C} 9-\mathrm{C} 10-\mathrm{C} 11$ & $178.0(2)$ \\
\hline $\mathrm{C} 12-\mathrm{N} 1-\mathrm{N} 2-\mathrm{N} 3$ & $-178.86(19)$ & $\mathrm{C} 8-\mathrm{C} 9-\mathrm{C} 10-\mathrm{C} 11$ & $-0.1(4)$ \\
\hline $\mathrm{N} 1-\mathrm{N} 2-\mathrm{N} 3-\mathrm{C} 4$ & $-0.3(3)$ & $\mathrm{C} 9-\mathrm{C} 10-\mathrm{C} 11-\mathrm{C} 6$ & $-0.7(4)$ \\
\hline $\mathrm{N} 2-\mathrm{N} 3-\mathrm{C} 4-\mathrm{C} 5$ & $0.6(3)$ & $\mathrm{C} 7-\mathrm{C} 6-\mathrm{C} 11-\mathrm{C} 10$ & $1.0(3)$ \\
\hline $\mathrm{N} 2-\mathrm{N} 3-\mathrm{C} 4-\mathrm{C} 6$ & $179.3(2)$ & $\mathrm{C} 4-\mathrm{C} 6-\mathrm{C} 11-\mathrm{C} 10$ & $-179.9(2)$ \\
\hline $\mathrm{N} 2-\mathrm{N} 1-\mathrm{C} 5-\mathrm{C} 4$ & $0.5(2)$ & $\mathrm{C} 5-\mathrm{N} 1-\mathrm{C} 12-\mathrm{C} 13$ & $150.9(2)$ \\
\hline $\mathrm{C} 12-\mathrm{N} 1-\mathrm{C} 5-\mathrm{C} 4$ & $179.1(2)$ & $\mathrm{N} 2-\mathrm{N} 1-\mathrm{C} 12-\mathrm{C} 13$ & $-30.7(3)$ \\
\hline
\end{tabular}




$\begin{array}{llll}\mathrm{N} 3-\mathrm{C} 4-\mathrm{C} 5-\mathrm{N} 1 & -0.7(2) & \mathrm{C} 5-\mathrm{N} 1-\mathrm{C} 12-\mathrm{C} 17 & -30.0(3) \\ \mathrm{C} 6-\mathrm{C} 4-\mathrm{C} 5-\mathrm{N} 1 & -179.1(2) & \mathrm{N} 2-\mathrm{N} 1-\mathrm{C} 12-\mathrm{C} 17 & 148.5(2) \\ \mathrm{C} 5-\mathrm{C} 4-\mathrm{C} 6-\mathrm{C} 7 & 156.9(2) & \mathrm{C} 17-\mathrm{C} 12-\mathrm{C} 13-\mathrm{C} 14 & -1.6(4) \\ \mathrm{N} 3-\mathrm{C} 4-\mathrm{C} 6-\mathrm{C} 7 & -21.4(3) & \mathrm{N} 1-\mathrm{C} 12-\mathrm{C} 13-\mathrm{C} 14 & 177.6(2) \\ \mathrm{C} 5-\mathrm{C} 4-\mathrm{C} 6-\mathrm{C} 11 & -22.2(4) & \mathrm{C} 12-\mathrm{C} 13-\mathrm{C} 14-\mathrm{C} 15 & 0.6(4) \\ \mathrm{N} 3-\mathrm{C} 4-\mathrm{C} 6-\mathrm{C} 11 & 159.5(2) & \mathrm{C} 13-\mathrm{C} 14-\mathrm{C} 15-\mathrm{C} 16 & -179.1(2) \\ \mathrm{C} 11-\mathrm{C} 6-\mathrm{C} 7-\mathrm{C} 8 & -0.4(3) & \mathrm{F} 1-\mathrm{C} 15-\mathrm{C} 16-\mathrm{C} 17 & 0.8(4) \\ \mathrm{C} 4-\mathrm{C} 6-\mathrm{C} 7-\mathrm{C} 8 & -179.5(2) & \mathrm{C} 14-\mathrm{C} 15-\mathrm{C} 16-\mathrm{C} 17 & -1.2(4) \\ \mathrm{C} 6-\mathrm{C} 7-\mathrm{C} 8-\mathrm{C} 9 & -0.4(4) & \mathrm{C} 15-\mathrm{C} 16-\mathrm{C} 17-\mathrm{C} 12 & 0.1(4) \\ \mathrm{C} 7-\mathrm{C} 8-\mathrm{C} 9-\mathrm{O} 9 & -177.6(2) & \mathrm{C} 13-\mathrm{C} 12-\mathrm{C} 17-\mathrm{C} 16 & 1.2(4) \\ \mathrm{C} 7-\mathrm{C} 8-\mathrm{C} 9-\mathrm{C} 10 & 0.7(4) & \mathrm{N} 1-\mathrm{C} 12-\mathrm{C} 17-\mathrm{C} 16 & -177.9(2) \\ \mathrm{C} 10-\mathrm{C} 9-\mathrm{O} 9-\mathrm{C} 18 & 14.0(4) & & \end{array}$

